\title{
Ketamine use for endotracheal intubation in severe sepsis and septic shock
}

\author{
SEOK WOO JO, SUNG YEON HWANG, IK JOON JO, TAE RIM LEE, HEE \\ YOON, WON CHUL CHA, MIN SEOB SIM, TAE GUN SHIN
}

Department of Emergency Medicine, Samsung Medical Center, Sungkyunkwan University School of Medicine, Seoul

* Seok Woo Jo and Sung Yeon Hwang contributed equally to this work

Corresponding author:

Tae Gun Shin

Department of Emergency Medicine

Samsung Medical Center

Sungkyunkwan University School of Medicine

81 Irwon-ro, Gangnam-gu, Seoul 135-710, Korea

Phone: +82-2-3410-2053

Fax: +82-2-3410-0012

E-mail:tackles@naver.com

\section{ABSTRACT}

Objective. We conducted this study to evaluate the clinical outcomes of patients with severe sepsis and septic shock who were treated with ketamine for endotracheal intubation.

Methods. A single-center, retrospective study was carried out to compare the outcomes of patients with severe sepsis and septic shock who received a ketamine or non-ketamine agent for rapid sequence intubation (RSI). We analyzed the sepsis registry for adult patients who presented to the emergency department (ED), met the criteria for severe sepsis or septic shock, and underwent endotracheal intubation between August 2008 and March 2014. The primary outcome was 28 -day mortality. We performed a multivariable logistic regression analysis to assess the association between ketamine use for intubation and 28-day mortality.

Results. In all, 170 patients were intubated during the study period. Of the eligible patients, 95 received ketamine and 75 received a non-ketamine agent. The 28-day mortality of the ketamine group was not significantly different from that of the nonketamine group ( $38 \%$ vs. $40 \%$, respectively, $\mathrm{P}=0.78)$. The unadjusted odds ratio (OR) of ketamine use for 28-day mortality was 0.92 (95\% CI: $0.49-1.70, \mathrm{P}=0.78$ ). The association remained insignificant after adjusting for age, gender, malignancy, initial lactate level on ED admission, time to first antibiotic administration, Acute Physiology and Chronic Health Evaluation II score on admission day, and propensity score regarding ketamine use (adjusted OR: 1.09; 95\% confidence interval [CI]: 0.49-2.40;
$\mathrm{P}=0.84$ ). Initial serum lactate on ED admission was the only significant predictive factor of 28-day mortality (adjusted OR: 1.23; 95\% CI: $1.10-1.38$; $\mathrm{P}<0.01)$.

Conclusions. For patients with severe sepsis and septic shock who were intubated using RSI, we found no significant difference in 28-day mortality between those who received ketamine as a sedative agent and those who received alternative sedatives.

Key words: sepsis, ketamine, intubation, mortality

\section{INTRODUCTION}

Severe sepsis and septic shock are lifethreatening conditions frequently complicated by airway compromise and acute respiratory failure. According to recent large studies, approximately $30 \%-95 \%$ of critically ill patients with sepsis require intubation. (1-5) Endotracheal intubation with mechanical ventilation is a life-saving intervention used in such situations to support the increased work of breathing, improve oxygenation and ventilation, and secure a patent airway.

Several studies have demonstrated that the rapid sequence intubation (RSI) technique, in which a potent sedative agent, followed by a rapidly acting neuromuscular blocking agent administered to facilitate endotracheal intubation, provides optimal intubating conditions while minimizing untoward complications even in emergency airway situations. $(6,7)$ However, the choice of appropriate sedatives for RSI in critically ill patients with sepsis is a chal- lenging task. Such patients are likely to have limited physiologic reserves and might be very sensitive to sedatives; even low doses of sedatives can have a deleterious effect on patient hemodynamics. Among several sedatives, etomidate has been widely used for RSI in critically ill patients because of its predictable onset and duration of action and minimal effects on hemodynamic indices. However, concerns have been raised regarding potential detrimental effects of etomidate-related adrenal insufficiency in septic patients, although the causal relationship between adrenal insufficiency and outcome remains inconclusive. (8)

Ketamine has received renewed attention in the emergency airway arena because it shares similar pharmacologic features (except adrenal suppression) with etomidate. (9) Although the current opinion is that ketamine is a good alternative to etomidate for intubation in critically ill patients with sepsis, few studies have specifically investigated the outcomes of patients with severe sepsis or septic shock treated with ketamine. (10) Therefore, we conducted this study to evaluate the clinical outcomes of patients with severe sepsis or septic shock who received ketamine during endotracheal intubation.

\section{METHODS}

A retrospective, single-center cohort study of patients with severe sepsis or septic shock who were intubated using the RSI technique was conducted at Samsung Medical Center (a 1,960-bed, universityaffiliated tertiary teaching hospital), located in a metropolitan city. The study 
was approved by the Institutional Review Board, and informed consent was waived because it was a retrospective study and no intervention was performed.

\section{Patient inclusion criteria}

Patients who met all of the following criteria were included for analysis: 1) 18 years of age or older; 2) those who presented to the emergency department (ED) with severe sepsis with serum lactate concentration $\geq 4 \mathrm{mmol} / \mathrm{l}$ or septic shock; and 3) patients who underwent RSI within 72 hours of ED administration. The decision to intubate was based on clinical evidence of respiratory distress (e.g., the use of accessory muscles during respiration) and/ or altered mental status requiring airway protection. The selection of intubating devices and medications was entirely dependent on the preferences of the operator. After verification of proper tube placement, post-intubation management, including applying the ventilator, was conducted according to standard protocol.

\section{Definition}

The definition of sepsis used in this study was defined by the American College of Chest Physicians/Society of Critical Care Medicine Consensus Conference. (11) Severe sepsis was described as sepsis associated with acute organ dysfunction. Septic shock was defined as sepsis that presented with hypotension (systolic blood pressure $<90 \mathrm{mmHg}$, mean arterial pressure [MAP] $<60 \mathrm{mmHg}$, or a reduction in systolic blood pressure $>40 \mathrm{mmHg}$ from baseline) despite adequate fluid resuscitation (30 $\mathrm{ml} / \mathrm{kg}$ ) in the absence of other causes of hypotension.

Body mass index (BMI) was used to classify patients as underweight (BMI $<18.5$ $\mathrm{kg} / \mathrm{m} 2)$, normal weight $(18.5 \leq \mathrm{BMI}<25$ $\mathrm{kg} / \mathrm{m} 2$ ), or overweight (BMI $\geq 25 \mathrm{~kg} / \mathrm{m} 2$ ). Time to antibiotic administration was defined as the time from ED arrival until the initiation of the first antibiotic administration.

\section{Data collection and outcome measures}

Data on patients who met the criteria for severe sepsis or septic shock on ED arrival were prospectively collected from August 2008 to March 2014, including every intubation attempt performed in the ED and intensive care unit (ICU). The data had been previously used in other studies of severe sepsis and septic shock. (12-14) The protocol for the management of patients with severe sepsis or septic shock was based on the protocol by Rivers et al. and international guidelines (2008 and 2012 Surviving Sepsis Campaign (SSC) Guidelines). Patients were treated according to the protocol, and the process was supervised by a board-certified emergency physician or intensivist. The following data were retrieved from a sepsis registry and electronic medical records: general characteristics of patients, including gender, age, body mass index, and comorbidity, as well as laboratory data including initial serum lactate, source of infection, and sepsis interventions such as fluid management. We also collected data related to the intubation procedure as follows: physiologic measures before intubation, drug administration for RSI, and vasopressor and corticosteroid use. The Sequential Organ Failure Assessment (SOFA) score and the Acute Physiology and Chronic Health Evaluation (APACHE) II score at baseline were calculated at the time of diagnosis of severe sepsis or septic shock to determine the estimated disease severity.

The primary outcome of the study was 28 day mortality. The secondary outcomes were overall in-hospital length of stay (LOS), ICU LOS, ventilator days, and inhospital mortality.

\section{Statistical analysis}

The included patients were categorized into either a ketamine group (those who received ketamine) or a non-ketamine group (those who received non-ketamine agents, such as etomidate or midazolam) based on the type of sedative agent used for RSI. Data are presented as the median with interquartile range (IQR) for numeric data and as number with percentage for categorical data. The Wilcoxon rank sum test was used for continuous variables, while the Chi-square test was employed for categorical variables.

Multiple logistic regression models were constructed to predict ketamine use for endotracheal intubation. In these models, , we adjusted the variable for different baseline characteristics including presence of malignancy, initial mean arterial pressure, any vasopressor use before intubation, time to antibiotic administration, location of intubation, and reasons for intubation. In addition, other multiple logistic regression models were constructed to assess the association between ketamine use and 28-day mortality. Two models were developed. Model 1 was adjusted for age, gender, malignancy, initial lactate level, time to antibiotic administration and severity of illness as assessed by the APACHE II score. In Model 2, the propensity score regarding ketamine use was added in addition to the adjusting factors used in Model 1. Initial MAP, time to antibiotic administration, any vasopressor use before intubation and location of intubation were used for calculating the propensity score. The goodness-of-fit of the final logistic regression model was assessed using the Hosmer-Lemeshow test and Nagelkerke's R2. Area under the receiver operating characteristic (AUC) curve was used to indicate classification accuracy. The results were presented as the adjusted odds ratio (OR) with a $95 \%$ confidence interval (CI). A P-value less than 0.05 was considered statistically significant. STATA 13.0 (STATA Corporation, College Station, TX, USA) was used for statistical analyses.

\section{RESULTS}

\section{Comparison of baseline characteristics}

A total of 170 patients were intubated during the study period. Of the eligible patients, 95 received ketamine and 75 received a non-ketamine agent. The sedative agents used in the non-ketamine group were etomidate $(\mathrm{n}=40)$ and midazolam $(n=35)$. The baseline characteristics of each group are described in table 1. Age, gender, BMI and suspected infection focus were not different between the groups. Patients with malignancy presented more frequently in the ketamine group than in the non-ketamine group. The ketamine group showed a significantly lower MAP on ED arrival compared to the non-ketamine group. Accordingly, any vasopressor use before intubation was significantly higher in the ketamine group than it was in the non-ketamine group (60\% vs. $35 \%$ respectively, $\mathrm{P}<0.01)$. Initial serum lactate and APACHE II scores were not related to the type of sedative agent used.

\section{Variables associated with intubation}

A comparison of the variables associated with intubation is shown in table 2. Immediately before intubation, vital signs, including MAP, heart rate, respiratory rate and peripheral capillary oxygen saturation were not different between the two groups. The median time to intubate following ED admission was 6.9 (IQR: 2.6 - 22.5) hours and was not significantly different between the two groups $(\mathrm{P}=0.14)$. Respiratory failure was the most frequent reason for intubation in both groups. Overall, succi- 
Table 1. Comparison of baseline characteristics.

\begin{tabular}{|c|c|c|c|c|}
\hline & $\begin{array}{l}\text { Overall group } \\
(n=170)\end{array}$ & $\begin{array}{l}\text { Ketamine group } \\
(\mathbf{n}=95)\end{array}$ & $\begin{array}{l}\text { Non-ketamine group } \\
(\mathbf{n}=75)\end{array}$ & P-value \\
\hline Age (years), median [IQ] & $67(55-74)$ & $67(57-73)$ & $68(53-75)$ & 0.86 \\
\hline Gender (male), n (\%) & $103(61)$ & $60(63)$ & $43(57)$ & 0.44 \\
\hline BMI (kg/cm2), n (\%) & & & & 0.73 \\
\hline Under & $29(17)$ & $18(19)$ & $11(15)$ & \\
\hline Normal & $103(61)$ & $57(60)$ & $46(61)$ & \\
\hline Over & $38(22)$ & $20(21)$ & $18(24)$ & \\
\hline \multicolumn{5}{|l|}{ Comorbidities, n (\%) } \\
\hline Hypertension & $56(33)$ & $31(33)$ & $25(33)$ & 0.92 \\
\hline Diabetes & $21(12)$ & $13(14)$ & $8(11)$ & 0.55 \\
\hline Cardiovascular disease & $20(12)$ & $9(9)$ & $11(15)$ & 0.30 \\
\hline Chronic lung disease & $13(8)$ & $7(7)$ & $6(8)$ & 0.88 \\
\hline Chronic renal disease & $6(4)$ & $2(2)$ & $4(5)$ & 0.26 \\
\hline Chronic hepatic disease & $9(5)$ & $3(3)$ & $6(8)$ & 0.16 \\
\hline Malignancy ${ }^{\star}$ & $92(54)$ & $59(62)$ & $33(44)$ & 0.02 \\
\hline Infection focus, $\mathrm{n}(\%)$ & & & & 0.92 \\
\hline Intra-abdominal & $35(21)$ & $20(21)$ & $15(20)$ & \\
\hline Respiratory & $98(58)$ & $55(58)$ & $43(57)$ & \\
\hline Urinary tract & $9(5)$ & $4(4)$ & $5(7)$ & \\
\hline Wound/Soft tissue & $10(6)$ & $6(6)$ & $4(5)$ & \\
\hline CNS & $1(1)$ & $0(0)$ & $1(1)$ & \\
\hline Indwelling device & $3(2)$ & $2(2)$ & $1(1)$ & \\
\hline Other infections & $14(8)$ & $8(8)$ & $6(8)$ & \\
\hline \multicolumn{5}{|l|}{ Initial vital signs, median [IQ] } \\
\hline Mean arterial pressure $(\mathrm{mmHg})$ & $66(56-82)$ & $61(55-77)$ & $69(58-93)$ & 0.02 \\
\hline Heart rate (beats per minute) & $121(102-138)$ & $119(105-137)$ & $121(99-140)$ & 0.76 \\
\hline Respiratory rate (breaths per minute) & $24(20-32)$ & $22(20-32)$ & $24(20-32)$ & 0.55 \\
\hline Body temperature $\left({ }^{\circ} \mathrm{C}\right)$ & $37.7(36.5-38.7)$ & $38.0(36.9-38.8)$ & $37.4(36.4-38.6)$ & 0.17 \\
\hline Initial serum lactate (mmol/l), median [IQ] & $5.1(3.5-7.1)$ & $4.8(3.3-7.0)$ & $5.3(3.7-7.8)$ & 0.21 \\
\hline SOFA score, median [IQ] & $9(6-11)$ & $9(6-11)$ & $9(6-11)$ & 0.70 \\
\hline APACHE II score, median [IQ] & $20(16-25)$ & $20(17-25)$ & $20(15-25)$ & 0.69 \\
\hline Time to antibiotic ${ }^{\star}(\mathrm{min})$, median [IQ] & $132(91-187)$ & $125(83-171)$ & $148(95-224)$ & $<0.01$ \\
\hline Steroid use, n (\%) & $84(49)$ & $52(55)$ & $32(43)$ & 0.12 \\
\hline Any vasopressor use before intubation*, n (\%) & $83(49)$ & $57(60)$ & $26(35)$ & $<0.01$ \\
\hline Vasopressor use within the first 7 days after admission & $132(78)$ & $74(78)$ & $58(73)$ & 0.93 \\
\hline
\end{tabular}

Data are shown as the median with interquartile range or as $\mathrm{n}(\%)$.

P-values were calculated using the Wilcoxon rank sum test or the Pearson's chi-square test.

${ }^{*}$ P-value less than 0.05

ALT, alanine aminotransferase; APACHE II score, Acute Physiology and Chronic Health Evaluation II score; AST, aspartate aminotransferase; INR, international normalized ratio; PT, prothrombin time; SOFA, Sequential Organ Failure Assessment.

nylcholine was the most commonly used neuromuscular blocking agent during RSI, and it produced no significant differences between the two groups.

\section{Comparison of outcomes}

The overall 28 -day mortality was $39 \%$, as shown in table 3 . The 28-day mortality of the ketamine group was not significantly different from that of the non-ketamine group ( $38 \%$ vs. $40 \%$, respectively; $\mathrm{P}=0.78$ ). The in-hospital LOS (ketamine group vs. non-ketamine group, 17 vs. 11 days; $\mathrm{P}=0.19$ ) as well as the ICU LOS (ketamine group vs. non-ketamine group, 4 vs. 4 days;
$\mathrm{P}=0.80$ ) were not significantly different between the two groups. In addition, there was no significant difference between the two groups regarding the number of ventilator days (ketamine group vs. non-ketamine group, 5 vs. 5 days; $\mathrm{P}=0.28$ ). 
Table 2. Variables associated with intubation.

\begin{tabular}{|c|c|c|c|c|}
\hline & $\begin{array}{l}\text { Overall group } \\
(n=170)\end{array}$ & $\begin{array}{l}\text { Ketamine group } \\
(\mathrm{n}=95)\end{array}$ & \multicolumn{2}{|c|}{$\begin{array}{l}\text { Non-ketamine group P-value } \\
(\mathrm{n}=75)\end{array}$} \\
\hline \multicolumn{5}{|c|}{ Vital signs and SPO2 before intubation, median [IQR] } \\
\hline Mean arterial pressure $(\mathrm{mmHg})$ & $77(65-93)$ & $77(65-91)$ & $78(65-95)$ & 0.58 \\
\hline Heart rate (beats per minute) & $122(110-137)$ & $121(110-135)$ & $123(108-140)$ & 0.48 \\
\hline Respiratory rate (breaths per minute) & $29(24-35)$ & $30(25-35)$ & $28(22-35)$ & 0.32 \\
\hline $\mathrm{SPO} 2(\%)$ & $92(87-96)$ & $93(88-97)$ & $90(86-96)$ & 0.17 \\
\hline Time to intubation (hour), median [IQR] & $7.9(3.1-23.2)$ & $7.6(3.6-25.9)$ & $8.2(1.5-22.1)$ & 0.31 \\
\hline Reasons for intubation*, n (\%) & & & & $<0.01$ \\
\hline Altered mental status & $28(16)$ & $4(4)$ & $24(32)$ & \\
\hline Respiratory failure & $126(74)$ & $79(83)$ & $47(62)$ & \\
\hline Shock & $16(9)$ & $12(13)$ & $4(5)$ & \\
\hline Location of intubation*, n (\%) & & & & $<0.01$ \\
\hline ED & $86(51)$ & $37(39)$ & $49(65)$ & \\
\hline ICU & $84(49)$ & $58(61)$ & $26(35)$ & \\
\hline Neuromuscular blocking agents, n (\%) & & & & 0.55 \\
\hline Succinylcholine & $124(73)$ & $71(75)$ & $53(71)$ & \\
\hline Others $\dagger$ & $46(27)$ & $24(25)$ & $22(29)$ & \\
\hline
\end{tabular}

Data are shown as the median with interquartile range or $\mathrm{n}(\%)$.

P-values were calculated using the Wilcoxon rank sum test or the Pearson's chi-square test.

$\mathrm{ED}$, emergency department; ICU, intensive care unit.

${ }^{*}$ P-value less than 0.05

†Others: Vecuronium, Cisacuratriumracy percent was 77\%, and area under the receiver operating characteristic curve for predicting ketamine use was 0.79 .

CI, confidence interval; ED, emergency department; ICU, intensive care unit; OR, odds ratio.

Table 3. Clinical outcomes.

\begin{tabular}{lllll}
\hline & $\begin{array}{l}\text { Overall group } \\
(\mathbf{n = 1 7 0 )}\end{array}$ & $\begin{array}{l}\text { Ketamine group } \\
(\mathbf{n}=\mathbf{9 5})\end{array}$ & $\begin{array}{l}\text { Non-ketamine group P-value } \\
(\mathbf{n}=\mathbf{7 5})\end{array}$ \\
\hline 28-day mortality, n (\%) & $66(39)$ & $36(38)$ & $30(40)$ & 0.78 \\
\hline In-hospital mortality, n (\%) & $79(46)$ & $43(45)$ & $36(48)$ & 0.72 \\
\hline In-hospital LOS (days), median [IQR] & $15(4-29)$ & $17(7-30)$ & $11(4-27)$ & 0.19 \\
\hline ICU LOS (day), median [IQR] & $4(2-8)$ & $4(2-8)$ & $4(2-6)$ & 0.80 \\
\hline Ventilator days, median [IQR] & $5(3-10)$ & $5(3-11)$ & $5(3-9)$ & 0.28 \\
\hline Total vasopressor administration time (hour), median [IQR] & $28(1-61)$ & $31(1-64)$ & $26(7-56)$ & 0.67 \\
\hline
\end{tabular}

Data are shown as the median with interquartile range or $\mathrm{n}(\%)$.

P-values were calculated using the Wilcoxon rank sum test or the Pearson's chi-square test.

ICU, intensive care unit; LOS, length of stay.

\section{Multivariable analysis}

The results of multivariable analysis to predict ketamine use for endotracheal intubation are shown in table 4. Adjusted OR of initial mean arterial pressure was 0.98 (95\% CI 0.960-0.996; $\mathrm{P}=0.02$ ). Adjusted ORs of respiratory failure and shock were 10.08 (95\% CI 3.02-33.68; $\mathrm{P}<0.01)$ and 14.65 (95\% CI 2.93-73.22; $\mathrm{P}<0.01$ ), respectively, compared to altered mental status.

Multivariable analysis models to assess as- sociations between ketamine use for intubation and 28-day mortality are shown in table 5. The unadjusted OR of ketamine use for 28-day mortality was 0.92 (95\% CI: $0.49-$ $1.70 ; \mathrm{P}=0.78)$. The association remained non-significant after adjusting for patient age, patient gender, malignancy, initial lactate level on ED admission, time to first antibiotic administration and APACHE II score on admission day (adjusted OR: 1.01; 95\% CI: 0.51-2.01; $\mathrm{P}=0.97$ ) (Model 1). The final models included the adjusting factors used in Model 1 and the propensity score regarding ketamine use. This model also demonstrated that ketamine use to facilitate intubation was not associated with 28-day mortality (adjusted OR: 1.09; 95\% CI: 0.492.40; $\mathrm{P}=0.84$ ) (Model 2). The initial serum lactate value on $\mathrm{ED}$ admission was the only significant predicting factor of 28-day mortality (adjusted OR: 1.23; 95\% CI: 1.10-1.38; $\mathrm{P}<0.01)$. 
Table 4. Multivariable analysis to predict ketamine use for endotracheal intubation in patients with severe sepsis or septic shock.

\begin{tabular}{llll}
\hline & OR & $\mathbf{9 5 \%} \mathbf{C I}$ & P-value \\
\hline Malignancy & 1.69 & $0.82-3.48$ & 0.15 \\
\hline Initial mean arterial pressure & 0.98 & $0.960-0.996$ & 0.02 \\
\hline Any vasopressor use before intubation & 1.62 & $0.72-3.65$ & 0.24 \\
\hline Time to antibiotic administration & 0.88 & $0.75-1.04$ & 0.13 \\
\hline Location of intubation & & \\
\hline ED & Reference & & \\
\hline ICU & 0.55 & $0.25-1.22$ & 0.14 \\
\hline Reasons for intubation & & \\
\hline Altered mental status & Reference & & \\
\hline Respiratory failure* & 10.08 & $3.02-33.68$ & $<0.01$ \\
\hline Shock $^{*}$ & 14.65 & $2.93-73.22$ & $<0.01$ \\
\hline
\end{tabular}

${ }^{*}$ P-value less than 0.05

$\dagger$ Time from emergency department arrival until initiation of the first antibiotic administration

The goodness-of-fit of the model was 0.23 with the Hosmer-Lemeshow test and 0.34 with Nagelkerke's R2. Classification accu

Table 5. Associations between ketamine use for intubation and 28-day mortality in patients with severe sepsis or septic shock after adjustments for potential confounding factors.

\begin{tabular}{llll}
\hline & OR & 95\% CI & P-value \\
\hline Model 1 & & & \\
\hline Ketamine use & 1.01 & $0.51-2.01$ & 0.97 \\
\hline Patient age & 1.00 & $0.98-1.03$ & 0.99 \\
\hline Patient gender & 0.98 & $0.49-1.96$ & 0.96 \\
\hline Malignancy & 1.90 & $0.92-3.89$ & 0.08 \\
\hline Initial lactate level $^{\star}$ & 1.23 & $1.10-1.37$ & $<0.01$ \\
\hline Time to antibiotic administration $\dagger$ & 1.07 & $0.95-1.20$ & 0.28 \\
\hline Initial APACHE II score & 0.99 & $0.95-1.04$ & 0.83 \\
\hline Model 2 & & & \\
\hline Ketamine use & 1.09 & $0.49-2.40$ & 0.84 \\
\hline Patient age & 1.00 & $0.97-1.02$ & 0.95 \\
\hline Patient gender & 0.98 & $0.49-1.95$ & 0.95 \\
\hline Malignancy & 1.98 & $0.93-4.23$ & 0.08 \\
\hline Initial lactate level ${ }^{\star}$ & 1.23 & $1.10-1.38$ & $<0.01$ \\
\hline Time to antibiotic administration $\dagger$ & 1.06 & $0.94-1.19$ & 0.37 \\
\hline Initial APACHE II score & 0.99 & $0.95-1.04$ & 0.81 \\
\hline Propensity score regarding ketamine use & 0.74 & $0.14-4.03$ & 0.73 \\
\hline
\end{tabular}

${ }^{*}$ P-value less than 0.05

$\dagger$ Time from emergency department arrival until initiation of the first antibiotic administration

The goodness-of-fit of the final model I was 0.34 with the Hosmer-Lemeshow test and 0.15 with Nagelkerke's R2. Classification accuracy percent was $72 \%$, and area under the receiver operating characteristic curve (AUROC) for predicting 28-day mortality of Model I was 0.67 .

The goodness-of-fit of the final model II was 0.31 with the Hosmer-Lemeshow test and 0.15 Nagelkerke's R2. Classification accuracy percent was $71 \%$, and AUROC for predicting 28-day mortality of Model II was 0.68 .

APACHE II score, Acute Physiology and Chronic Health Evaluation II score; CI, confidence interval; OR, odds ratio. 


\section{DISCUSSION}

In this study, we observed that ketamine use for RSI in patients with severe sepsis or septic shock did not influence 28-day mortality compared to alternative agents. Furthermore, we found no significant differences in any secondary measures, including ventilator days, ICU LOS, hospital LOS and in-hospital mortality, according to the use of any sedatives.

Ketamine is the only currently available sedative comparable to etomidate that is associated with favorable hemodynamic profiles. Several studies have demonstrated the usefulness of ketamine as a sedative agent in many different fields. Patanwala et al. investigated first-pass success intubation in patients who received etomidate or ketamine for RSI performed in the ED. They showed that first-pass success intubation was equivalent despite any use of ketamine or etomidate in the ED. However, outcomes regarding morbidity or mortality were not described. (15) Ballow et al. developed a standardized RSI medication protocol for trauma patients who require ED intubation at a level I trauma center. (16) Ketamine was incorporated into the protocol as a sedative agent for RSI. The authors concluded that the use of ketamine was effective in critically injured patients, even those with traumatic brain injury. Price et al. assessed the hemodynamics before and after ketamine use for prehospital intubation in the air medical setting. In their study, ketamine use was not associated with any adverse hemodynamic parameter changes compared to etomidate. (17) Although ketamine has been used as a main intubation drug for many years, there are discrepancies between real world practices and supporting evidence for the use of ketamine for RSI in septic patients with hemodynamic instability. Only a few studies have specifically investigated the impact of ketamine on outcome in such septic patients. Jabre et al. prospectively evaluated 655 critically ill patients, including injured, stroke, and septic patients, who received either etomidate or ketamine for intubation. They showed that a single dose of etomidate was not associated with increased morbidity or mortality compared to ketamine. In a subgroup analysis of septic patients $(n=76)$, there were also no significant differences in outcomes between etomidate and ketamine use. Jabre et al. suggested that ketamine is safe and offers a good alternative to etomidate for intubation in septic patients. However, they also stated that this finding could only be attributed to their small number of included septic patients. Our data provide additional evidence to support the use of ketamine in patients with severe sepsis or septic shock. In patients with severe sepsis or septic shock, ketamine use as a part of RSI has not been shown to increase 28-day mortality compared to those who received alternative sedatives. Other outcomes, including ventilator days, ICU LOS and in-hospital LOS, which are important outcomes associated with preventing hospitalacquired infections and reducing hospital costs, were also not different according to the use of any sedatives.

Ketamine has potential advantages over alternative sedatives, which makes it attractive for patients with severe sepsis or septic shock. First, ketamine does not affect adrenal function, which is the main drawback of etomidate use in septic patients. Second, ketamine increases catecholamine concentrations and stimulates the sympathetic nervous system; as a result, it increases blood pressure and systemic vascular resistance in patients without catecholamine depletion. As septic patients are at greater risk of hemodynamic deterioration, this characteristic might be a valuable property. However, one should be cautious, as the direct negative inotropic effects of ketamine on the myocardium might outweigh the indirect sympathetic stimulating effects in patients with severely prolonged shock or catecholamine depletion, resulting in hemodynamic deterioration or even cardiac arrest. (18) Finally, in vitro and animal studies have suggested that ketamine acts as a protective modulator of proinflammatory cytokines, such as TNF-a, IL-1 $\beta$, and IL-6. $(19,20)$ A previous limited study performed in post-operative patients also showed that ketamine inhibited serum IL-6 and superoxide anions produced by neutrophils. $(21,22)$ However, the impact of these properties of ketamine on the outcome of septic patients is still not confirmed and well-designed studies will be required to establish the clear role of ketamine in septic patients.
The results of a multiple logistic regression analysis in this study revealed that elevated serum lactate on ED admission was the only significant factor that predicted 28day mortality in patients with severe sepsis or septic shock who were intubated using RSI. Despite its limitations, serum lactate has been widely used in the field of sepsis care, such as for identifying tissue hypoperfusion and assessing the severity of sepsis and resuscitation endpoints. $(14,23,24)$ Our finding reinforces the importance of the early measurement of serum lactate, as recommended by international guidelines. (25) This parameter will help clinicians identify patients at higher risk for death; we suggest providing a more aggressive management to reduce the mortality in septic patients with an elevated serum lactate level on ED arrival.

There are several limitations to this study that should be considered. First, it was a retrospective,single-center study and is therefore limited to other settings in its generalizability. Second, the selection of drugs was entirely dependent on the operator's preference, which might have caused a selection bias. Third, the study population included patients with severe sepsis or septic shock, producing heterogeneity in the patient characteristics. However, we only selected patients with severe sepsis who had a serum lactate level greater than $4 \mathrm{mmol} / \mathrm{l}$, which indicates cryptic shock. Puskarich et al. showed that mortality in severe sepsis with an elevated serum lactate ( $\geq 4 \mathrm{mmol} / \mathrm{l}$ ) was comparable to that of overt septic shock. (26) Recently published data from the Surviving Sepsis Campaign database also indicated that patients with a lactate level greater than $4 \mathrm{mmol} / \mathrm{l}$ were significantly associated with in-hospital mortality regardless of the presence of hypotension. (27)

\section{CONCLUSION}

For patients with severe sepsis or septic shock who were intubated using RSI, we found no significant difference in 28-day mortality between those who received ketamine as a sedative agent and those who received alternative sedatives.

\section{REFERENCES}

1. Annane D, Vignon P, Renault A, Bollaert PE, Charpentier C, Martin C, et al. Norepinephrine plus dobutamine versus epinephrine alone for management of septic shock: a randomised trial. Lancet 2007;370(9588):676-84.

2. Sakles JC, Mosier J, Patanwala AE, Dicken J. Learning curves for direct laryngoscopy and GlideScope(R) video laryngoscopy in an 
emergency medicine residency. Simul Healthc 2014;9(6):377-83. doi: 10.1097/SIH.0000000000000057.

3. ProCESS Investigators. A Randomized Trial of Protocol-Based Care for Early Septic Shock. N Engl J Med . 2014;370(18):1683-93.

4. Mouncey PR, Osborn TM, Power GS, Harrison DA, Sadique MZ, Grieve RD, et al. Trial of Early, Goal-Directed Resuscitation for Septic Shock. N Engl J Med 2015;372(14):1301-11.

5. Caironi P, Tognoni G, Masson S, Fumagalli R, Pesenti A, Romero M, et al. Albumin Replacement in Patients with Severe Sepsis or Septic Shock. N Engl J Med 2014;370(15):1412-21.

6. Li J, Murphy-Lavoie H, Bugas C, Martinez J, Preston C. Complications of emergency intubation with and without paralysis. Am J Emerg Med 1999;17(2):141-3.

7. Sagarin MJ, Barton ED, Chng YM, Walls RM. Airway management by US and Canadian emergency medicine residents: a multicenter analysis of more than 6,000 endotracheal intubation attempts. Ann Emerg Med. 2005;46(4):328-36.

8. Mohammad Z, Afessa B, Finkielman JD. The incidence of relative adrenal insufficiency in patients with septic shock after the administration of etomidate. Crit Care 2006;10(4):R105.

9. Pai A, Heining M. Ketamine. Continuing Education in Anaesthesia, Critical Care \& Pain. 2007;7(2):59-63.

10. Jabre P, Combes X, Lapostolle F, Dhaouadi M, Ricard-Hibon A, Vivien B, et al. Etomidate versus ketamine for rapid sequence intubation in acutely ill patients: a multicentre randomised controlled trial. Lancet 2009;374(9686):293-300.

11. Bone RC, Balk RA, Cerra FB, Dellinger RP, Fein AM, Knaus WA, et al. Definitions for sepsis and organ failure and guidelines for the use of innovative therapies in sepsis. The ACCP/SCCM Consensus Conference Committee. American College of Chest Physicians/Society of Critical Care Medicine. Chest 1992;101(6):1644-55.

12. Shin TG, Jo IJ, Choi DJ, Kang MJ, Jeon K, Suh GY, et al. The adverse effect of emergency department crowding on compliance with the resuscitation bundle in the management of severe sepsis and septic shock. Crit Care 2013;17(5):R224.

13. Kang MJ, Shin TG, Jo IJ, Jeon K, Suh GY, Sim MS, et al. Factors influencing compliance with early resuscitation bundle in the management of severe sepsis and septic shock. Shock (Augusta, Ga) 2012;38(5):474-9.

14. Hwang SY, Shin TG, Jo IJ, Jeon K, Suh GY, Lee TR, et al. Association between hemodynamic presentation and outcome in sepsis patients. Shock (Augusta, Ga) 2014;42(3):205-10.

15. Patanwala AE, McKinney CB, Erstad BL, Sakles JC. Retrospective analysis of etomidate versus ketamine for first-pass intubation success in an academic emergency department. Acad Emerg Med 2014;21(1):87-91.

16. Ballow SL, Kaups KL, Anderson S, Chang M. A standardized rapid sequence intubation protocol facilitates airway management in critically injured patients. J Trauma Acute Care Surg 2012;73(6):1401-5.

17. Price B, Arthur AO, Brunko M, Frantz P, Dickson JO, Judge T, et al. Hemodynamic consequences of ketamine vs etomidate for endotracheal intubation in the air medical setting. Am J Emerg Med 2013;31(7):1124-32.

18. Dewhirst E, Frazier WJ, Leder M, Fraser DD, Tobias JD. Cardiac arrest following ketamine administration for rapid sequence intubation. J Intensive Care Med 2013;28(6):375-9.

19. Chang Y, Chen TL, Sheu JR, Chen RM. Suppressive effects of ketamine on macrophage functions. Toxicol Appl Pharmacol 2005;204(1):27-35.

20. Wu GJ, Chen TL, Ueng YF, Chen RM. Ketamine inhibits tumor necrosis factor-alpha and interleukin-6 gene expressions in lipopolysaccharide-stimulated macrophages through suppression of toll-like receptor 4-mediated c-Jun N-terminal kinase phosphorylation and activator protein-1 activation. Toxicol Appl Pharmacol 2008;228(1):105-13.

21. Roytblat L, Talmor D, Rachinsky M, Greemberg L, Pekar A, Appelbaum A, et al. Ketamine attenuates the interleukin-6 response after cardiopulmonary bypass. Anesth Analg 1998;87(2):266-71.

22. Zilberstein G, Levy R, Rachinsky M, Fisher A, Greemberg L, Shapira Y, et al. Ketamine attenuates neutrophil activation after cardiopulmonary bypass. Anesth Analg 2002;95(3):531-6, table of contents.

23. Shapiro NI, Howell MD, Talmor D, Nathanson LA, Lisbon A, Wolfe RE, et al. Serum lactate as a predictor of mortality in emergency department patients with infection. Ann Emerg Med 2005;45(5):524-8.

24. Jones AE, Shapiro NI, Trzeciak S, Arnold RC, Claremont HA, Kline JA. Lactate clearance vs central venous oxygen saturation as goals of early sepsis therapy: a randomized clinical trial. JAMA . 2010;303(8):739-46.

25. Dellinger RP, Levy MM, Rhodes A, Annane D, Gerlach H, Opal SM, et al. Surviving sepsis campaign: international guidelines for management of severe sepsis and septic shock: 2012. Intensive Care Med 2013;41(2):580-637.

26. Puskarich MA, Trzeciak S, Shapiro NI, Heffner AC, Kline JA, Jones AE. Outcomes of patients undergoing early sepsis resuscitation for cryptic shock compared with overt shock. Resuscitation 2011;82(10):1289-93.

27. Casserly B, Phillips GS, Schorr C, Dellinger RP, Townsend SR, Osborn TM, et al. Lactate measurements in sepsis-induced tissue hypoperfusion: results from the Surviving Sepsis Campaign database. Crit Care Med 2015;43(3):567-73 